\title{
Voronoi neighbor statistics of hard-disks and hard-spheres
}

\author{
V. Senthil Kumar and V. Kumaran ${ }^{\text {a) }}$ \\ Department of Chemical Engineering, Indian Institute of Science, Bangalore 560 012, India
}

\begin{abstract}
The neighbor distribution in hard-sphere and hard-disk fluids is analyzed using Voronoi tessellation. The statistical measures analyzed are the $n$th neighbor coordination number $\left(C_{n}\right)$, the $n$th neighbor distance distribution $\left[f_{n}(r)\right]$, and the distribution of the number of Voronoi faces $\left(P_{n}\right)$. These statistics are sensitive indicators of microstructure, and they distinguish thermodynamic and annealed structures. A sharp rise in the hexagon population marks the onset of hard-disk freezing transition, and $C_{n}$ decreases sharply to the hexagonal lattice values. In hard-disk random structures the pentagon and heptagon populations remain significant even at high volume fraction. In dense hard-sphere (three-dimensional) structures at the freezing transition, $C_{1}$ is close to 14 , instead of the value of 12 expected for a face-centered-cubic lattice. This is found to be because of a topological instability, where a slight perturbation of the positions in the centers of a pair of particles transforms a vertex in the Voronoi polyhedron into a Voronoi surface. We demonstrate that the pair distribution function and the equation-of-state obtained from Voronoi tessellation are equal to those obtained from thermodynamic calculations. In hard-sphere random structures, the dodecahedron population decreases with increasing density. To demonstrate the utility of the neighbor analysis, we estimate the effective hard-sphere diameter of the Lennard-Jones fluid by identifying the diameter of the spheres in the hard-sphere fluid which has $C_{1}$ equal to that for the Lennard-Jones fluid. The estimates are within $2 \%$ deviation from the theoretical results of Barker-Henderson and Weeks-Chandler-Andersen.
\end{abstract}

\section{INTRODUCTION}

The Voronoi polyhedron of a nucleus point in space is the smallest polyhedron formed by the perpendicularly bisecting planes between the given nucleus and all the other nuclei. ${ }^{1}$ The Voronoi tessellation divides a region into spacefilling, nonoverlapping convex polyhedra. The salient properties of Voronoi tessellation are the following:

- Any point inside a Voronoi cell is closer to its nucleus than any other nuclei (Fig. 1). These cells are space filling and hence provide a precise definition of local volume. $^{2}$

- It gives a definition of geometric neighbors. The nuclei sharing a common Voronoi surface are geometric neighbors. Points on the shared surface are equidistant to the corresponding pair of nuclei. Hence, geometric neighbors are competing centers in a growth scenario.

- Voronoi cells of hard-spheres are irregular at lower packing fractions but become regular as the regular close packing is approached. Thus, they are useful in characterizing all structures from random to regular.

These properties qualify Voronoi tessellation as an important tool in the structural analysis of random media such as glass, packings, foams, cellular solids, proteins, etc. ${ }^{3-5}$ Voronoi tessellation occurs naturally in growth processes such as crystallization and plant cell growth. ${ }^{6}$ The statistical

${ }^{a)}$ Electronic mail: kumaran@chemeng.iisc.ernet.in distributions of many Voronoi cell properties are reported (see Zhu et al., ${ }^{7}$ Oger et al., ${ }^{8}$ and references therein). In this analysis, we use the Voronoi neighbor statistics to characterize the thermodynamic and annealed microstructures of harddisks and hard-spheres.

Section II presents the hard-core packing fractions of interest in this work. Section III introduces the Voronoi neighbor statistics. Let a central sphere's geometric neighbors be called first neighbors, i.e., the first layer of neighbors. The first neighbors' neighbors (which are themselves not first

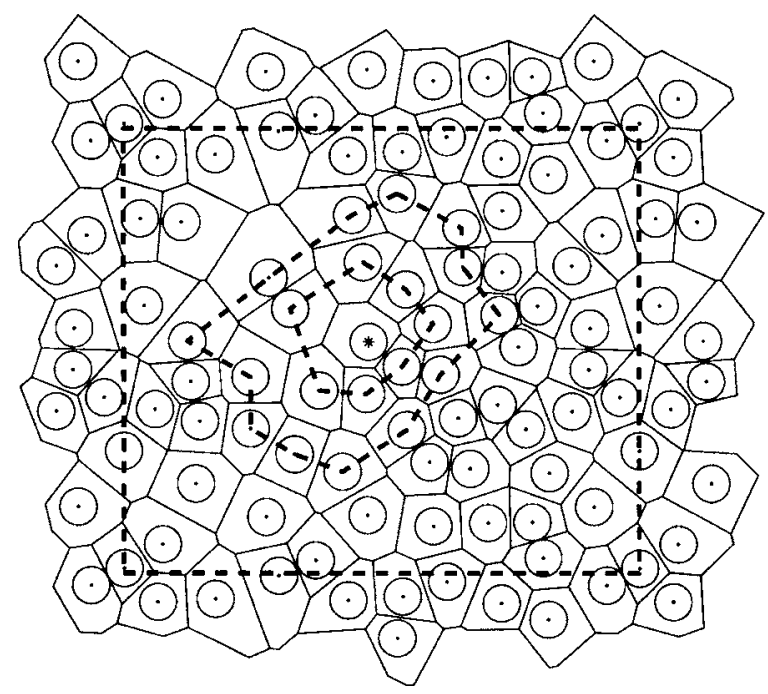

FIG. 1. The Voronoi tessellation of a hard-disk configuration, with periodic boundary conditions. Central box shown in dashed lines. The first and second neighbors of a central disk (*) are shown linked in dashed lines. 
TABLE I. Salient packing fractions in hard-rod, hard-disk, and hard-sphere systems.

\begin{tabular}{lccc}
\hline \hline & Hard-rod & Hard-disk & Hard-sphere \\
\hline$\sigma$ is & Length & Diameter & Diameter \\
Volume of the particle, $v_{p}$ & $\sigma$ & $\pi \sigma^{2} / 4$ & $\pi \sigma^{3} / 6$ \\
Cell volume at regular close packing $v_{c}$ & $\sigma$ & $\sqrt{3} \sigma^{2} / 2$ & $\sigma^{3} / \sqrt{2}$ \\
Freezing packing fraction, $\nu_{F}$ & $\ldots$ & $\approx 0.691^{\mathrm{a}}$ & $\approx 0.494^{\mathrm{b}}$ \\
Melting packing fraction, $\nu_{M}$ & $\ldots$ & $\approx 0.716^{\mathrm{a}}$ & $\approx 0.545^{\mathrm{b}}$ \\
Loose random packing, $\nu_{\text {LRP }}$ & $\ldots$ & $0.772 \pm 0.002^{\mathrm{c}}$ & $0.555 \pm 0.005^{\mathrm{d}}$ \\
Dense random packing, $\nu_{\text {DRP }}$ & $\ldots$ & $0.82 \pm 0.02^{\mathrm{e}}$ & $0.64 \pm 0.02^{\mathrm{e}}$ \\
Regular close packing, $\nu_{c}$ & 1 & $\pi /(2 \sqrt{3})$ & $\pi /(3 \sqrt{2})$ \\
\hline \hline
\end{tabular}

${ }^{\mathrm{a}}$ From Alder and Wainwright (Ref. 16).

${ }^{\mathrm{b}}$ From Hoover and Ree (Ref. 17).

${ }^{\mathrm{c}}$ From Hinrichsen et al. (Ref. 19).

${ }^{\mathrm{d}}$ From Onoda and Liniger (Ref. 15).

${ }^{\mathrm{e}}$ From Berryman (Ref. 18).

neighbors) are the second neighbors, and so on (Fig. 1). Thus, all the spheres surrounding a central sphere can be partitioned layerwise and characterized by $n$th neighbor coordination number $\left(C_{n}\right)$ and $n$th neighbor distance distribution function $\left[f_{n}(r)\right]$. In Sec. III it is shown that the information contained in the radial distribution function $[g(r)]$ can be partitioned into these sets of neighbor statistics. The distribution of the number of Voronoi bounding surfaces $\left(P_{n}\right)$ is also of interest because $C_{1}$ is its mean. These neighbor statistics are sensitive microstructural indicators, and they distinguish the thermodynamic and annealed structures. For a hard-rod system in one dimension, these neighbor statistics are exactly known, given in Sec. IV. For hard-disk and hardsphere systems, we review the neighbor statistics reported in literature and report $C_{n}$ and $P_{n}$ for the $N V E$ Monte Carlo (MC) and annealed configurations in Secs. V and VI.

We have generated the annealed structures by repeated cycles of swelling and random displacements, this is a MC adaptation of Woodcock's algorithm. ${ }^{9}$ The low-density annealed structures are identical to the thermodynamic structures. The dense annealed structures are quite distinct from the thermodynamic structures and are presumed to terminate at the dense random packing. However, as shown by Torquato et al., ${ }^{10}$ when there are inhomogeneities in the system consisting of crystallite domains in a dense random structure, it is possible to generate configurations denser than the dense random packing. The $C_{1}$ for random hard-sphere structures produced by this algorithm agrees well with the dense random packing experimental results of Finney. ${ }^{11}$

A sharp rise in the hexagon population marks the onset of hard-disk freezing; $C_{n}$ for $n>1$ decreases sharply to the hexagonal lattice values. In dense hard-disk random structures, the pentagon and heptagon populations remain significant even as the random close-packing limit is approached. In dense hard-disk structures, both thermodynamic and random, the number of pentagons and heptagons appear to be equal as the close-packing limit is approached. In dense hard-sphere (three-dimensional) structures at the freezing transition, $C_{1}$ is close to 14 , instead of the value of 12 expected for a face-centered-cubic lattice. This is found to be because of a topological instability analyzed by Troadec et al., ${ }^{12}$ where a slight perturbation of the positions in the centers of a pair of particles transforms a vertex in the
Voronoi polyhedron into a Voronoi surface. Due to topological instability, a slightly perturbed face-centered-cubic lattice of hard-spheres has Voronoi polyhedra with faces 12 to 18 , with the mean at 14 . Hence, on freezing transition the hardsphere $C_{1}$ is close to 14 rather than 12 . We demonstrate that this result is consistent with thermodynamic data. In hardsphere random structures, the dodecahedron population decreases with increasing density.

The notion of effective hard-sphere diameter for dense soft potential fluids has been extensively analyzed, see the recent review by Silva et al. ${ }^{13}$ It is known, both through simulations and experiments, that the structure of a dense soft potential fluid is nearly identical to that of the hardsphere fluid having a particular diameter. This diameter is the effective hard-sphere diameter. In Sec. VII, we show that using the equality of $C_{1}$ of the Lennard-Jones fluid to the hard-sphere fluid at some packing fraction, it is possible to estimate the effective hard-sphere diameter to within $2 \%$ deviation from the theoretical results of Barker-Henderson ${ }^{37}$ and Weeks-Chandler-Andersen ${ }^{38}$ and its modification by Lado. ${ }^{14}$

\section{HARD-CORE-SYSTEM PROPERTIES}

Let $v_{p}$ be the volume of the hard-sphere, $\rho$ the number density, and $v=1 / \rho$ the specific volume. The packing fraction is $\nu=v_{p} / v$. At regular close packing, let $v_{c}$ be the regular cell volume and $\nu_{c}=v_{p} / v_{c}$ the packing fraction. The normalized packing fraction is $y=\nu / \nu_{c}$. Other packing fractions of physical relevance are

- the freezing $\left(\nu_{F}\right)$ and melting $\left(\nu_{M}\right)$ packing fractions,

- the loose random packing $\left(\nu_{\mathrm{LRP}}\right)$ defined ${ }^{15}$ as the lowest-density isotropic packing that can support an infinitesimal external load at the limit of acceleration due to gravity tending to zero, and

- the dense random packing $\left(\nu_{\mathrm{DRP}}\right)$ defined as the highestdensity spatially homogeneous isotropic packing.

All these salient packing fractions are listed in Table I. There is no freezing transition for a hard-rod system. Also there are no random structures for hard-rods since the regular close- 


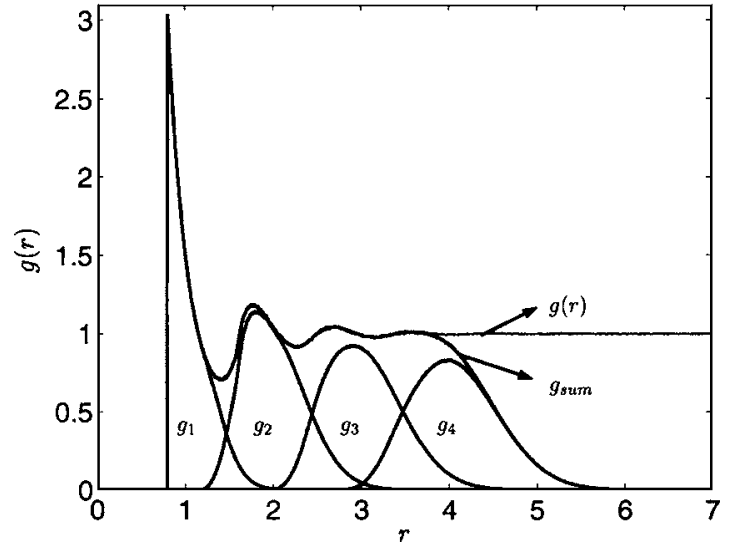

FIG. 2. Voronoi partitioning of hard-disk $g(r), \nu=0.50, g_{\text {sum }}=g_{1}+g_{2}+g_{3}$ $+g_{4}$. The $g_{n}(r)$ and $g_{\text {sum }}$ are shown in thick lines and $g(r)$ in thin line.

packed structure is the only load-bearing structure. For a Voronoi analysis of hard-disk loose random packing refer to Hinrichsen et al. ${ }^{19}$

\section{NEIGHBOR STATISTICS}

Let $N_{n}$ be the number of $n$th neighbors around a central sphere, then the $n$th neighbor coordination number is $C_{n}=\left\langle N_{n}\right\rangle$, where $\langle\cdot\rangle$ denotes the ensemble average. The radial distribution function is computed as

$$
g(r)=\frac{\rho(r)}{\rho}=\frac{1}{\rho} \frac{\left\langle\delta N_{r}\right\rangle}{\delta V_{r}}
$$

where $\delta V_{r}$ is the volume of the shell between $r$ and $r+d r$ around the central sphere and $\delta N_{r}$ is the number of spheres with their centers in the shell between $r$ and $r+d r$. The spheres around the central sphere can be partitioned layerwise as

$$
\delta N_{r}=\sum_{n=1}^{\infty} \delta N_{r}^{n}
$$

where $\delta N_{r}^{n}$ is the number of $n$th neighbors with their centers in the shell between $r$ and $r+d r$. Using Eq. (2) in Eq. (1), $g(r)$ can be partitioned as

$$
g(r)=\frac{1}{\rho_{n=1}} \sum_{n=1}^{\infty} \frac{\left\langle\delta N_{r}^{n}\right\rangle}{\delta V_{r}}=\sum_{n=1}^{\infty} g_{n}(r),
$$

where $g_{n}(r)$ is the $n$th neighbor radial distribution function. Figure 2 illustrates a Voronoi partitioning for hard-disk $g(r)$. Such a partitioning was first reported by $\operatorname{Rahman}^{20}$ and recently by Lavrik and Voloshin. ${ }^{21}$

The $n$th neighbor distance distribution function $f_{r}(r)$ is defined such that $f_{n}(r) d r$ is the fraction of the $n$th neighbors at a distance $r$ to $r+d r$, then,

$$
f_{n}(r) d r=\frac{\left\langle\delta N_{r}^{n}\right\rangle}{\left\langle\int_{0}^{\infty} \delta N_{r}^{n} d r\right\rangle}=\frac{\left\langle\delta N_{r}^{n}\right\rangle}{C_{n}} .
$$

Here, we have used $\left\langle\int_{0}^{\infty} \delta N_{r}^{n} d r\right\rangle=\left\langle N_{n}\right\rangle=C_{n}$. Using Eqs. (3) and (4), we get,

$$
g_{n}(r)=\frac{C_{n}}{\rho} \frac{f_{n}(r)}{\frac{\delta V_{r}}{d r}}=\frac{C_{n}}{\rho} \frac{f_{n}(r)}{S_{r}},
$$

where $S_{r}$ is the spherical surface area at $r, S_{r}=2$ in one dimension (1D), $S_{r}=2 \pi r$ in two dimensions (2D), and $S_{r}=4 \pi r^{2}$ in three dimensions (3D). Using Eq. (5) in Eq. (3), we get,

$$
g(r)=\frac{1}{\rho S_{r n=1}} \sum_{n}^{\infty} C_{n} f_{n}(r) .
$$

Equation (6) shows that the two Voronoi neighbor statistics, $C_{n}(\nu)$ and $f_{n}(r ; \nu)$, together contain the thermodynamic information in $g(r ; \nu)$. We consider another Voronoi statistic, the distribution of the number of bounding surfaces of the Voronoi cell, $P_{n}$. It is identical to the distribution of the number of the first neighbors, hence $C_{1}=\sum n P_{n}$. We will show in Secs. V and VI that these neighbor statistics are sensitive indicators of the microstructure which distinguish thermodynamic and annealed structures.

In hard-sphere systems the compressibility factor $Z=p /\left(\rho k_{B} T\right)$ is related to the radial distribution function at contact $g(\sigma)$ as

$$
Z=1+B_{2} \rho g(\sigma),
$$

where $B_{2}$ is the second virial coefficient, $B_{2}=\sigma$ for hard-rods, $B_{2}=(\pi / 2) \sigma^{2}$ for hard-disks, and $B_{2}=(2 / 3) \pi \sigma^{3}$ for hardspheres. Now $g(\sigma)=g_{1}(\sigma)$, since a sphere in contact is necessarily a first neighbor. Equation (5) gives $g_{1}(\sigma)=\left(C_{1} / \rho\right)\left[f_{1}(\sigma) / S_{\sigma}\right]$. Note that $B_{2} / S_{\sigma}=\sigma / 2 D$, where $D$ is the dimensionality of the system. Using these in Eq. (7) gives

$$
Z=1+\frac{\sigma}{2 D} C_{1} f_{1}(\sigma) .
$$

Thus, for the hard-sphere systems, the two neighbor statistic values $C_{1}$ and $f_{1}(\sigma)$ contain the thermodynamic information.

For any nondegenerate ${ }^{22}$ two-dimensional (2D) tessellation with periodic boundary conditions (PBC) or with a large number of particles, $C_{1}=6$ exactly. ${ }^{23,24}$ Using this in Eq. (8) for hard-disks we have $Z=1+\frac{3}{2} \sigma f_{1}(\sigma)$. This result was derived by Ogawa and Tanemura ${ }^{25}$ using a different but less general method, while the above derivation is valid for any dimensions and shows the role of $C_{1}$.

\section{HARD-ROD RESULTS}

For a hard-rod system $g_{n}(r)$ is exactly known, ${ }^{26}$

$$
g_{n}(r)= \begin{cases}0, & \text { if } r<n \sigma \\ \frac{v(r-n \sigma)^{n-1}}{(n-1) !(v-\sigma)^{n}} \exp \left(-\frac{r-n \sigma}{v-n \sigma}\right), & \text { if } r \geqslant n \sigma .\end{cases}
$$

Here, $S_{r}=2$ and $C_{n}=2$. Using this result in Eq. (5) gives $f_{n}(r)=(1 / v) g_{n}(r)$. Using this with Eq. (9) gives $f_{1}(\sigma)=1 /(v-\sigma)$. Using these results in Eq. (8) gives 


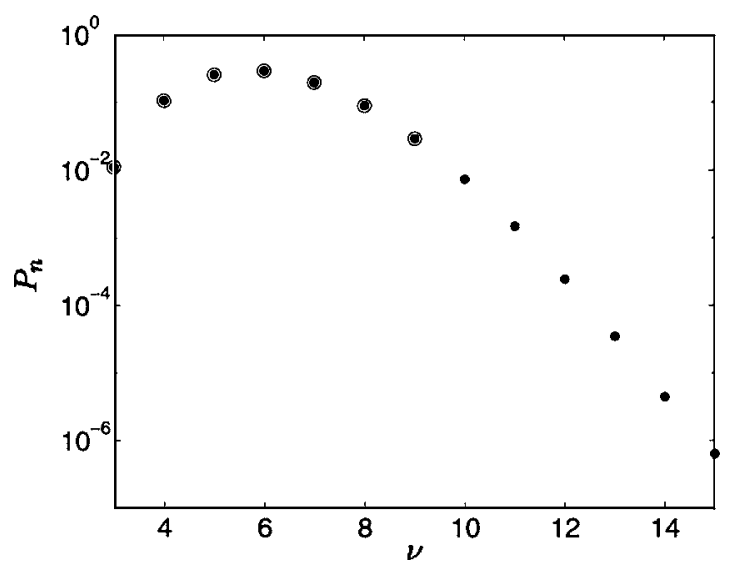

FIG. 3. $P_{n}$ for 2D Poisson tessellation. Theoretical result $(\bigcirc)$ from Calka (Ref. 30). Simulation data (๑) averaged for 3500 frames of 900 random points.

$$
Z=1+\frac{\sigma}{v-\sigma}=\frac{v}{v-\sigma}=\frac{1}{1-\nu} .
$$

This is the Tonks equation of state for hard-rods. ${ }^{27}$

For a hard-rod system $f_{n+1}(r)$ can be gotten from $f_{n}(r)$ exactly as

$$
f_{n+1}(r)=\int_{0}^{r} f_{1}\left(r-r^{\prime}\right) f_{n}\left(r^{\prime}\right) d r^{\prime}
$$

Such a simple convolution is not available for hard-disk and hard-sphere systems.

\section{HARD-DISK RESULTS}

For 2D Poisson tessellation $f_{1}(r)$ was derived by Collins, ${ }^{24}$

$$
f_{1}(r)=\frac{\pi \rho r}{3}\left[\rho^{1 / 2} r \exp \left(\frac{-\pi}{4} \rho r^{2}\right)+\operatorname{erfc}\left(\frac{\sqrt{\pi}}{2} \rho^{1 / 2} r\right)\right] .
$$

It was rederived by Stillinger et al $^{28}$ by a different method. Explicit expressions for 2D Poisson tessellation $P_{n}$ are available. ${ }^{29,30}$ We compare the Poisson $P_{n}$ data of Calka ${ }^{30}$ with our simulation results in Fig. 3.

We have studied two types of hard-disk structures: thermodynamic and swelled random structures. The thermodynamic structures are generated using NVE MC at 50\% success rate; i.e. the amplitude of the random trial displacement is adjusted such that $50 \%$ of the trials lead to nonoverlapping configurations. The swelled random structures are generated using a MC adaptation of the Woodcock's ${ }^{9}$ algorithm: swell all the particles till the nearest neighbors touch each other, give random trial displacements (with say $50 \%$ success rate as in NVE MC) for all the particles, and repeat the swelling and random displacements till the desired density is attained. The effect of the success rate on the randomness of the resultant structures is studied below.

As mentioned in Sec. III, for any nondegenerate 2D tessellation with PBC, $C_{1}=6$ exactly. Hence, $C_{1}$ is not a microstructural indicator for hard-disk structures. However, $C_{n}$, for $n>1$, are functions of $\nu$ and are sensitive indicators of mi-

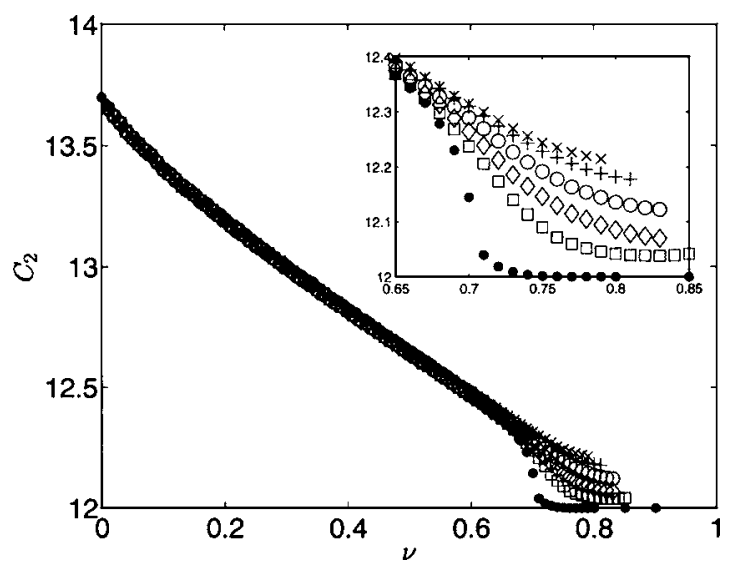

FIG. 4. $C_{2}$ for hard-disk $N V E(\bigcirc)$ and swelled random configurations at success rates of $10 \%(\square), 30 \%(\diamond), 50 \%(\bigcirc), 70 \%(+)$, and $90 \%(\times)$. NVE data averaged for 10000 configurations of 256 hard-disks. Swelled random data averaged for 1000 configurations of 256 hard-disks.

crostructure. $C_{2}$ and $C_{3}$ for hard-disk configurations are given in Figs. 4 and 5, from which we observe the following:

- For 2D Poisson tessellation $C_{2}^{0} \approx 13.698$ and $C_{3}^{0}$ $\approx 22.94$.

- Well below the freezing density, the swelled random structures are identical to the thermodynamic structures for any success rate. Above the freezing density, the thermodynamic structure $C_{n}(n>1)$ decreases sharply to the regular hexagonal lattice values $\left(C_{n}\right)_{\text {reg }}=6 n$, while the swelled random structure $C_{n}$ decreases slowly and nearly saturate at $\nu_{\mathrm{DRP}}$.

- If the swelled random configurations are generated with a low success rate, the large random trial displacements tend to equilibrate the local structures. However, if the success rate is high, the random trial displacements are small and the swelling process locks the particles into random structures. The Fig. 4 inset shows that as the success rate is lowered, the $C_{2}$ of the resultant structure gets closer to its thermodynamic value. The difference between the $C_{n}(n \neq 1$ in $2 \mathrm{D})$ of a given structure and that of the thermodynamic structure at the same density

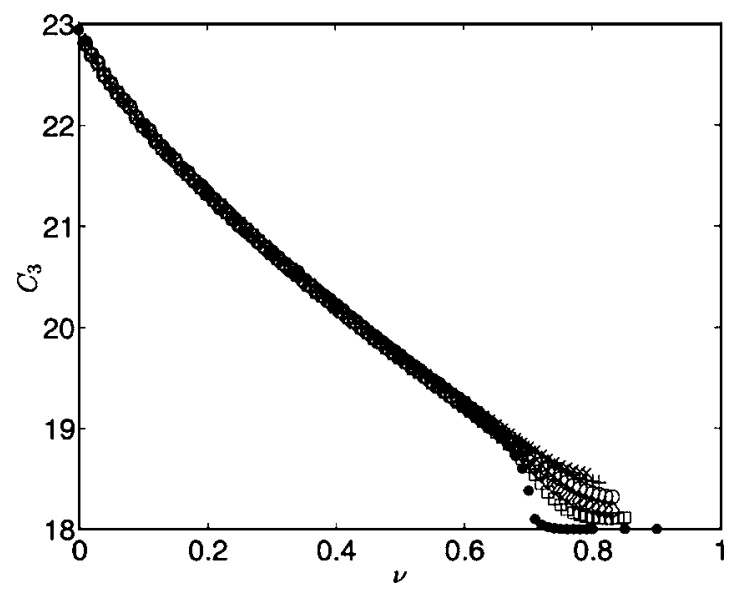

FIG. 5. $C_{3}$ for hard-disk $N V E(\bigcirc)$ and swelled random configurations at success rates of $10 \%(\square), 30 \%(\diamond), 50 \%(\bigcirc), 70 \%(+)$, and $90 \%(\times)$. Averaging as in Fig. 4. 


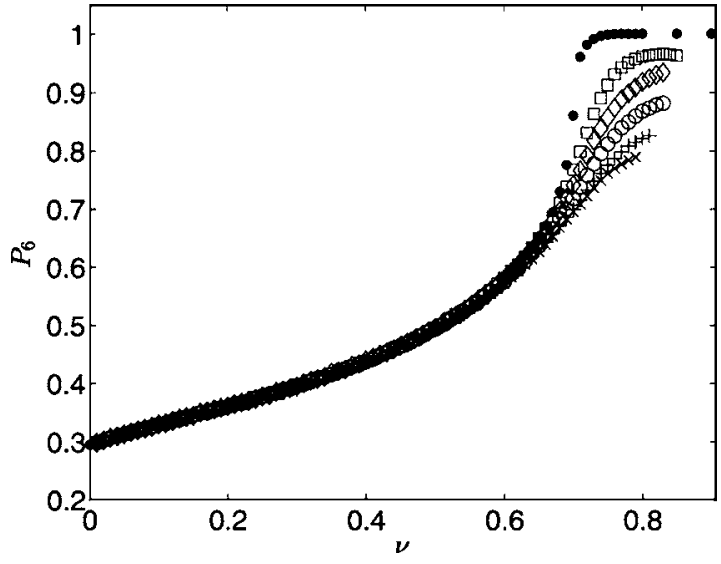

FIG. 6. $P_{6}$ for hard-disk $N V E(\bullet)$ and swelled random configurations at success rates of $10 \%(\square), 30 \%(\diamond), 50 \%(\bigcirc), 70 \%(+)$, and $90 \%(\times)$. Averaging as in Fig. 4.

is a measure of its randomness. The Fig. 4 inset also shows that for $\nu>\nu_{F}$ configurations with different degrees of randomness can be generated by tuning the success rates. The proximity of the $70 \%$ and $90 \%$ success rate structures in the said inset shows that the limiting case of near $100 \%$ success rate should give the maximally random structures. These structures should not sense the freezing transition, and hence their $C_{n}$ should not have an inflection point around the freezing density. From the inset, note that while the $10 \%$ success rate structures have an inflection about $\nu_{F}$, structures with success rates $50 \%$ and above show no visible inflection. The branch of maximally random structures is presumed to terminate at the dense random packing. However, by negotiating disorder with order, one can generate structures denser than the dense random packing. ${ }^{10}$ From the inset also, note that at $10 \%$ success rate, packing fractions as high as 0.85 are attainable, even though $\nu_{\mathrm{DRP}}=0.82 \pm 0.02$. This is possibly due to the formation of crystallite domains within the dense random structure. However, at $90 \%$ success rate, the maximum packing fraction attainable using the present algorithm does not exceed $\nu_{\mathrm{DRP}}$. It is interesting to note that the Voronoi neighbor statistics are sensitive even to the degree of randomness of the "random" structures.

Next, we study the number distribution of the Voronoi polygon edges, $P_{n}$. For 2D configurations with PBC, even though $C_{1}=\sum n P_{n}=6$ exactly, $P_{n}$ is a function of density and is a sensitive microstructural indicator. In Fig. 6 we compare the hexagon incidence in the swelled random structures for different success rates with that in the thermodynamic structures. However, for the incidence of other polygons, to avoid a profusion of figures, we compare only the thermodynamic structures (Fig. 7) and the 50\% success rate swelled random structures (Fig. 8). From these figures we observe the following:

- Figure 6 shows that, while the hexagon population rises sharply across the freezing transition for thermodynamic structures, it rises quite slowly for the random structures. As the success rate increases, the hexagon

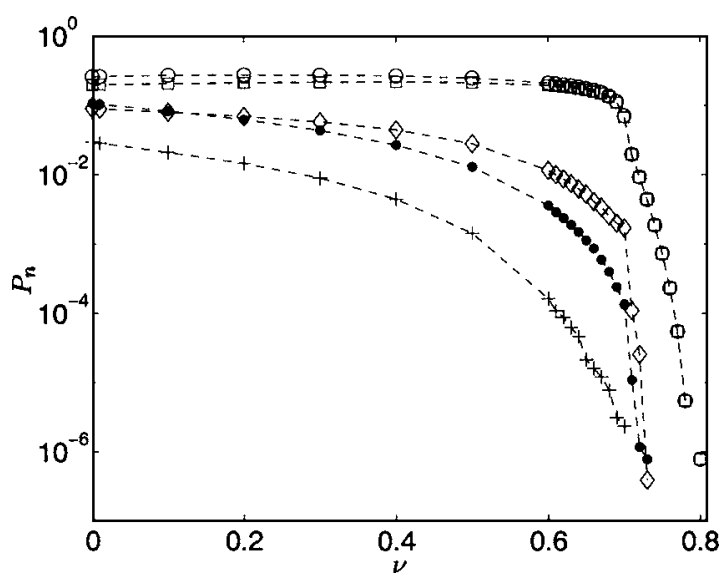

FIG. 7. $P_{n}$ for hard-disk $N V E$ configurations, for $n=4(\bullet), 5(\bigcirc), 7$ ( $\left.\square\right), 8$ $(\diamond)$, and $9(+)$. Averaging as in Fig. 4.

population at $\nu_{\mathrm{DRP}}$ decreases. This shows that increasing the success rate increases the randomness of the structures.

- From Fig. 7, for $\nu>\nu_{F}$ we see that the polygons, dominant after hexagons, are pentagons and heptagons. Also their populations are nearly identical. This population equality, also observed in the random structures (Fig. 8), may be explained as follows: In 2D structures with $\mathrm{PBC}$, if the populations of the polygons other than pentagons, hexagons, and heptagons are negligible (as in dense hard-disk structures), then the populations of pentagons and heptagons will be nearly identical, so that the mean number of sides is exactly six.

- From Fig. 8, it is seen that the pentagon and heptagon populations are quite significant in the dense random hard-disk structures.

- Polygons with faces 3, 10, 11, and 12 have sharply decreasing incidence as $\nu$ increases (even for $\nu<\nu_{F}$ ) in both thermodynamic and swelled random structures (figure not shown).

\section{HARD-SPHERE RESULTS}

For three-dimensional (3D) Poisson tessellation $C_{1}^{0}=\frac{48}{35} \pi^{2}+2 \approx 15.5354$, an exact result by Meijering. ${ }^{23}$ The

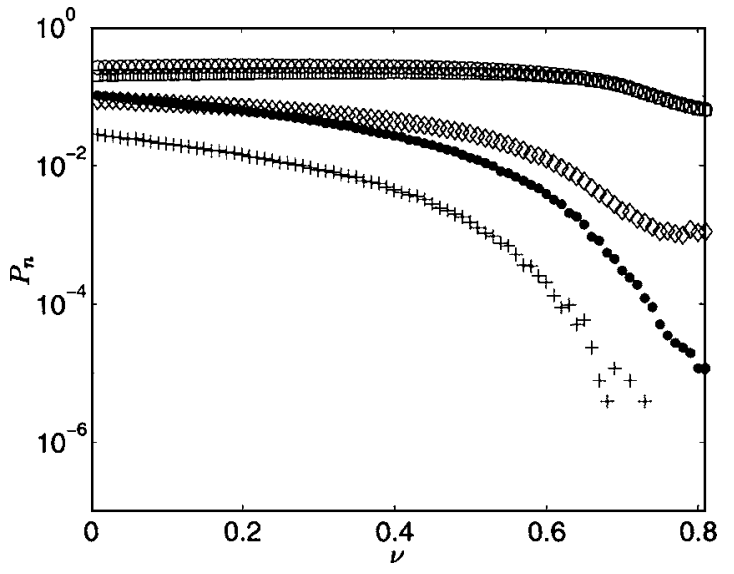

FIG. 8. $P_{n}$ for hard-disk $50 \%$ success rate swelled random configurations, for $n=4(\bullet), 5(\bigcirc), 7(\square), 8(\diamond)$, and $9(+)$. Averaging as in Fig. 4. 


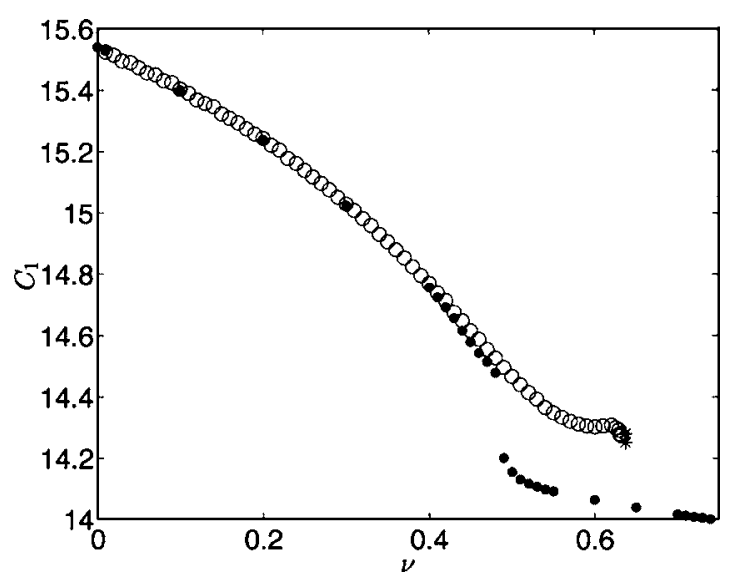

FIG. 9. $C_{1}$ for hard-sphere NVE (๑) vs $50 \%$ success rate swelled random (O) configurations and (*) are experimental results by Finney (Ref. 11). The NVE and swelled random data sets are averaged for 1000 configurations of 256 hard-spheres.

Wigner-Seitz or Voronoi cell for the perfect face-centeredcubic (fcc) lattice is the rhombic dodecahedron, and it has $C_{1}=12$ and $C_{2}=42$. Figures 9 and 10 respectively show $C_{1}$ and $C_{2}$ for thermodynamic and swelled random hard-sphere structures, from which we observe the following:

- For 3D Poisson tessellation $C_{2}^{0} \approx 69.8$.

- The sudden decrease of $C_{n}$ across the freezing transition is similar to that observed in the hard-disk system.

- Finney ${ }^{11}$ has reported $C_{1}$ for two different sets of experimental dense random packing configurations as $14.251 \pm 0.015$ and $14.28 \pm 0.05$. Figure 9 shows that the $C_{1}$ for random hard-sphere configurations match reasonably with the experimental results of Finney.

- Figure 9 shows that $C_{1}$ approaches 14 instead of the value of 12 expected for an fcc lattice. This is because a slight perturbation of the positions in the centers of a pair of particles transforms a vertex in the Voronoi polyhedron into a Voronoi surface, causing the coexistence of polyhedra with faces $12-18$, with the mean at 14 , as shown by Troadec et al. ${ }^{12}$ We briefly discuss this issue in the Appendix. $C_{1}$ increases from 12 to 14 by the

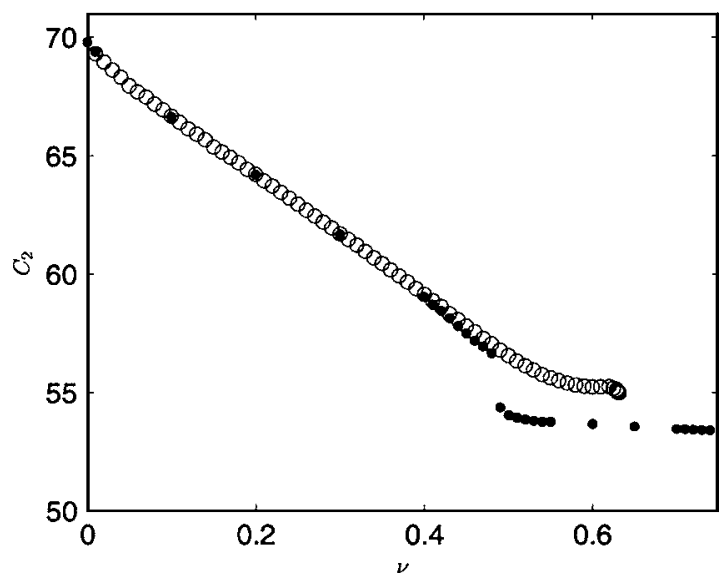

FIG. 10. $C_{2}$ for hard-sphere $N V E(\bigcirc)$ vs $50 \%$ success rate swelled random (○) configurations. Averaging as in Fig. 9.

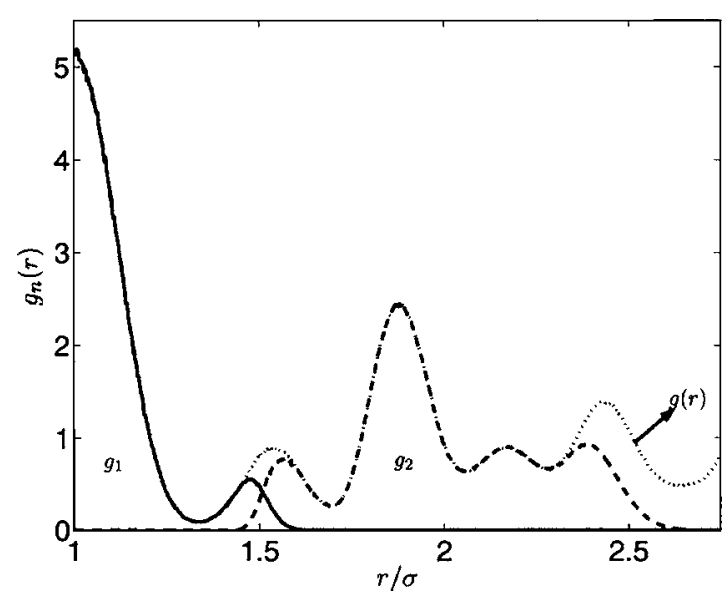

FIG. 11. $g_{n}(r)$ for thermodynamic hard-sphere configurations at $\nu=0.57$. The promotion of a few second neighbors into first neighbors manifests as a secondary peak in $g_{1}(r)$.

promotion of a few second neighbors into first neighbors, by the formation of additional tiny quadrilateral faces on the erstwhile rhombic dodecahedron (see Fig. 19). This promotion manifests as a secondary feature in $g_{1}(r)$ (Fig. 11) which grows as an inflection near $\nu_{F}$ and becomes a separate peak as $\nu$ increases.

TABLE II. System size/shape dependence and thermodynamic consistency checks for hard-sphere $C_{1}$.

\begin{tabular}{|c|c|c|c|c|c|}
\hline$\nu$ & $Z_{\mathrm{Hs}}{ }^{\mathrm{a}}$ & Run & $C_{1}$ & $f_{1}(\sigma)$ & $Z_{\text {vor }}^{\mathrm{b}}$ \\
\hline \multirow[t]{5}{*}{0.65} & \multirow{5}{*}{24.19} & $I^{\mathrm{c}}$ & 14.0390 & 9.96 & 24.31 \\
\hline & & II $^{\mathrm{d}}$ & 14.0388 & 9.99 & 24.38 \\
\hline & & $\mathrm{III}^{\mathrm{e}}$ & 14.0386 & 9.83 & 23.99 \\
\hline & & $\mathrm{IV}^{\mathrm{f}}$ & 14.0389 & 9.92 & 24.20 \\
\hline & & $\mathrm{V}^{\mathrm{g}}$ & 14.0382 & 9.93 & 24.24 \\
\hline \multirow[t]{5}{*}{0.68} & \multirow[t]{5}{*}{36.34} & I & 14.0252 & 15.00 & 36.07 \\
\hline & & II & 14.0260 & 15.13 & 36.37 \\
\hline & & III & 14.0251 & 15.30 & 36.77 \\
\hline & & IV & 14.0255 & 15.09 & 36.27 \\
\hline & & $\mathrm{V}$ & 14.0249 & 15.20 & 36.53 \\
\hline \multirow[t]{5}{*}{0.70} & \multirow[t]{5}{*}{54.47} & I & 14.0172 & 22.97 & 54.65 \\
\hline & & II & 14.0168 & 23.08 & 54.93 \\
\hline & & III & 14.0165 & 23.11 & 55.00 \\
\hline & & IV & 14.0164 & 23.16 & 55.10 \\
\hline & & $\mathrm{V}$ & 14.0175 & 22.97 & 54.67 \\
\hline \multirow[t]{5}{*}{0.72} & \multirow[t]{5}{*}{108.05} & I & 14.0083 & 46.23 & 108.94 \\
\hline & & II & 14.0080 & 46.15 & 108.74 \\
\hline & & III & 14.0083 & 46.67 & 109.95 \\
\hline & & IV & 14.0083 & 46.29 & 109.08 \\
\hline & & $\mathrm{V}$ & 14.0085 & 46.63 & 109.86 \\
\hline
\end{tabular}

${ }^{\mathrm{a}}$ Young and Alder (Ref. 31) give the hard-sphere solid equation of state as $Z=3 / \alpha+2.566+0.55 \alpha-1.19 \alpha^{2}+5.95 \alpha^{3}$, where $\alpha=\left(v-v_{c}\right) / v_{c}=(1-y) / y$ is the dimensionless excess free volume.

${ }^{\mathrm{b}}$ Using Eq. (8).

${ }^{c}$ Averaged for 1000 configurations of 256 hard-spheres in a cubic box, with PBC.

${ }^{\mathrm{d}}$ Averaged for 512 configurations of 500 hard-spheres in a cubic box.

${ }^{\mathrm{e}}$ Averaged for 500 configurations of 512 hard-spheres in a cuboidal box $\left(l_{x}: l_{y}: l_{z}=2: 1: 1\right)$.

${ }^{\mathrm{f}}$ Averaged for 297 configurations of 864 hard-spheres in a cubic box.

${ }^{\mathrm{g}}$ Averaged for 187 configurations of 1372 hard-spheres in a cubic box. 


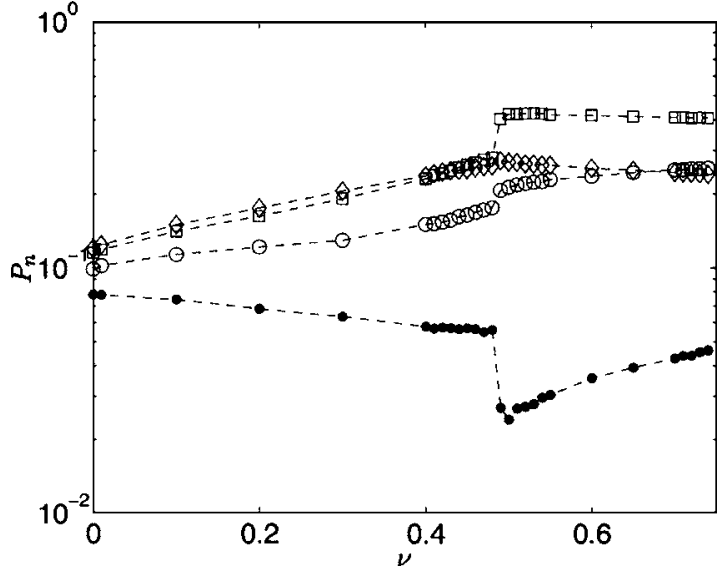

FIG. 12. $P_{n}$ for hard-sphere NVE configurations, for $n=12(\bullet), 13(\bigcirc), 14$ $(\square)$, and $15(\diamond)$. Averaging as in Fig. 9.

Table II shows the system size/shape dependence and thermodynamic consistency checks on the hard-sphere $C_{1}$ data. It shows that $C_{1}$ tending to 14 near regular close packing is consistent with the thermodynamic data. $C_{1}$ data shows negligible size dependence since it depends only on the enumeration of the first neighbors. For simulations in a cubical box with PBC, the number of spheres must be more than $(6 / \pi) \times\left(C_{1}+\cdots+C_{(n-1)}\right)$ to have meaningful averages for the higher order $C_{n}$. Table II also shows a comparison of the thermodynamic compressibility factor obtained from the hard-sphere equation of state (Young and Alder ${ }^{31}$ ) with the compressibility factor obtained from Eq. (8), in which $C_{1}$ and $f_{1}(\sigma)$ are determined by Voronoi tessellation. This table shows that the compressibility factor obtained from Voronoi tessellation is in agreement with the thermodynamic data, even though $C_{1}$ is larger than the value of 12 expected for a fcc lattice.

Next, we study the number distribution of the Voronoi polyhedra faces, $P_{n}$. We compare the data for the thermodynamic configurations in Figs. 12 and 13 with those for the 50\% success rate swelled random configurations in Figs. 14 and 15 . From these figures we observe the following:

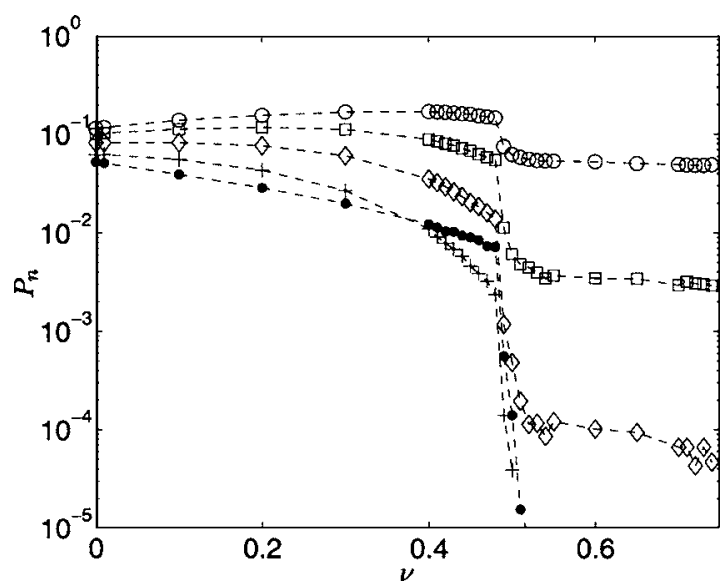

FIG. 13. $P_{n}$ for hard-sphere NVE configurations, for $n=11(\bullet), 16(\bigcirc), 17$ $(\square), 18(\diamond)$, and $19(+)$. Averaging as in Fig. 9.

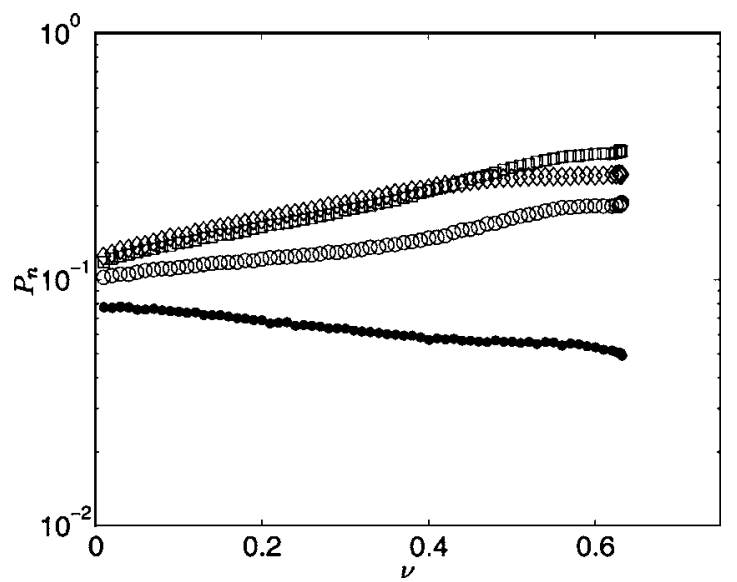

FIG. 14. $P_{n}$ for hard-sphere $50 \%$ success rate swelled random configurations, for $n=12(\bullet), 13(\bigcirc), 14(\square)$, and $15(\diamond)$. Averaging as in Fig. 9.

- A rise in the dodecahedron population marks the freezing transition. However, the populations of 13-18 faceted polyhedra remain significant even near $\nu_{c}$. It is also apparent that $P_{n}$ near close packing for equilibrium structures is not very different from that near the dense random packing for annealed structures due to the topological instability (Fig. 16). The $P_{n}$ data near dense random packing agrees well with that of Jullien et al. ${ }^{32}$

- Figure 14 shows that in the random hard-sphere structures, the dodecahedron population decreases with increasing $\nu$. This behavior is unlike that in the random hard-disk structures (Fig. 6), where the hexagon population steadily increases with $\nu$. The decrease in dodecahedron population cannot be interpreted as a decrease in fcc crystallites because a slightly perturbed fcc crystallite may get accounted for in the 13-18 faceted polyhedra population.

- Comparing the $P_{n}$ data in Figs. 13 and 15, we see that the population of the polyhedra with faces 11 and 16-19 decreases sharply across the freezing transition in the thermodynamic structures, but it decreases only gradually in the random structures.

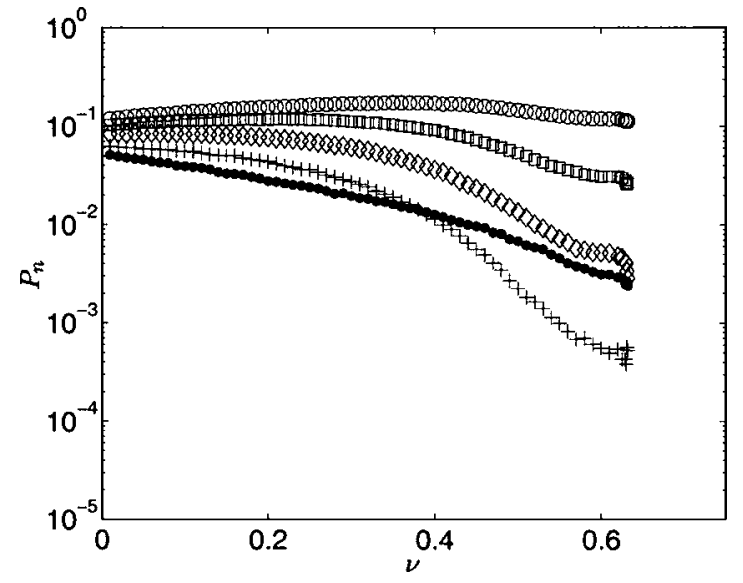

FIG. 15. $P_{n}$ for hard-sphere $50 \%$ success rate swelled random configurations, for $n=11(\bullet), 16(\bigcirc), 17(\square), 18(\diamond)$, and $19(+)$. Averaging as in Fig. 9. 


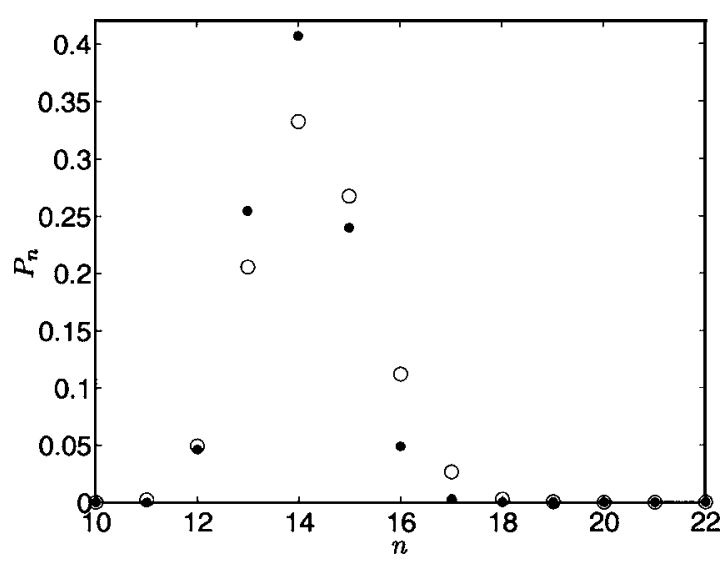

FIG. 16. Comparison of $P_{n}$ for a near regular close-packing thermodynamic structure at $\nu=0.74(\mathbf{O})$ and a near dense random packing swelled random structure at $\nu=0.632(\bigcirc)$.

A similar topological instability occurs for a simple cubic lattice in the hard-disk system, which has $C_{1}=4$. In $2 \mathrm{D}$ tessellations, vertices with four edges incident on them are topologically unstable and any slight perturbation of the lattice transforms a vertex into a Voronoi edge, resulting in $C_{1}=6$. However, since the regular close-packed structure in two dimensions is a hexagonal structure, in which the number of Voronoi edge is stable under a slight perturbation of the particle centers, this effect is not observed in two dimensions.

\section{ESTIMATION OF THE EFFECTIVE HARD-SPHERE DIAMETER FOR LENNARD-JONES FLUID FROM $C_{1}$}

The notion of effective hard-sphere diameter (EHSD) has a long history, beginning with Boltzmann. ${ }^{33} \mathrm{He}$ suggested that the distance of closest approach of the soft potential molecules could be considered the EHSD. ${ }^{33}$ Experiments and simulations have shown that the structure (characterized by the radial distribution function ${ }^{34}$ or its Fourier transform, the structure factor ${ }^{35}$ ) of the dense soft potential fluid can be matched with that of the hard-sphere fluid having a particular diameter. This defines the EHSD of the soft potential fluid at a given density and temperature. The EHSD method has been proven successful in the prediction of thermodynamic properties, self-diffusion coefficient, ${ }^{13}$ and shear viscosity ${ }^{36}$ of Lennard-Jones (LJ) fluids.

The LJ potential $\phi_{\mathrm{LJ}}(r)=4 \epsilon_{\mathrm{LJ}}\left[\left(\sigma_{\mathrm{LJ}} / r\right)^{12}-\left(\sigma_{\mathrm{LJ}} / r\right)^{6}\right]$ has an energy scale $\epsilon_{\mathrm{LJ}}$ and a length scale $\sigma_{\mathrm{LJ}}$ called the molecular diameter. The dimensionless temperature is $T^{*}=k_{B} T / \epsilon_{\mathrm{LJ}}$ and the dimensionless density is $\rho^{*}=\rho \sigma_{\mathrm{LJ}}^{3}$. The effective hard-sphere diameter $\sigma$ is rendered dimensionless as $\sigma^{*}=\sigma / \sigma_{\mathrm{LJ}}$. The theoretical approaches of Barker and Henderson $^{37}(\mathrm{BH})$ and of Chandler, Weeks, and Andersen ${ }^{38}$ (WCA) and their modification by Lado $^{14}$ (denoted here as LWCA) integrate the repulsive part of the LJ potential with different criteria yielding different expressions for the EHSDs. The explicit expressions for these models, as presented in Ben-Amotz and Herschbach, ${ }^{39}$ along with their empirical results (denoted here as $\mathrm{BAH}$ ) obtained by fitting the equation-of-state data to Carnahan-Starling-van der Waals equation are given in Table III.

We estimate the EHSD as follows: By Voronoi tessellating the LJ configurations we get $C_{1}$. The effective hardsphere packing fraction $\nu$ is gotten by interpolating the thermodynamic hard-sphere $C_{1}$ vs $\nu$ data (Fig. 9). Then, the dimensionless EHSD is computed as $\sigma^{*}=\left(6 \nu / \pi / \rho^{*}\right)^{1 / 3}$. This method of estimating the EHSD of the soft potential fluid requires that the averaged local neighborhood, as characterized by $C_{1}$, be identical with that of the hard-sphere fluid having the diameter $\sigma$, the EHSD. This method is based on the statistics of the geometry of particle distributions and is similar to Boltzmann's method ${ }^{33}$ which is based on the trajectories. Such statistical-geometric approaches are simpler because they do not use any integral criteria for the repulsive part of the potential (as in the BH, WCA, or LWCA models) or employ any property data fitting (as in the BAH model). Table IV shows ten different state points for the LJ fluid; the first five state points are in the liquid state, while the rest are in the gaseous state. For these state points, the values of EHSD predicted from $C_{1}$ show less than $2 \%$ deviation from the $\mathrm{BH}, \mathrm{WCA}$, and LWCA models and less than 5\% deviation from the $\mathrm{BAH}$ model. It may be noted that the deviations among these models is also of the same order (see, for example, Fig. 7 of Ben-Amotz and Herschbach ${ }^{39}$ ). The excellent match of the EHSD computed from the statisticalgeometric approach with those based on the integral criteria for the repulsive part of the potential (as in the BH, WCA, and LWCA models) shows the validity of the EHSD concept and also acts as a validation of the computational procedure used here.

\section{CONCLUSIONS}

We have analyzed the $n$th neighbor coordination number $\left(C_{n}\right)$, the $n$th neighbor distance distribution $\left[f_{n}(r)\right]$, and the distribution of the number of Voronoi faces $\left(P_{n}\right)$ for harddisk and hard-sphere systems for both thermodynamic and annealed structures. The annealed structures were produced by repeated cycles of swelling and random displacements, with the success rate of these random displacements being a

TABLE III. The parameters $\sigma_{0}^{*}$ and $T_{0}^{*}$ in $\sigma^{*}=\sigma_{0}^{*}\left[1+\left(T^{*} / T_{0}^{*}\right)^{1 / 2}\right]^{-1 / 6}$ for the different EHSD models, as presented in Ben-Amotz and Herschbach (Ref. 39).

\begin{tabular}{lcc}
\hline \hline \multicolumn{1}{c}{ Model } & $\sigma_{0}^{*}$ & $T_{0}^{*}$ \\
\hline Barker-Henderson (BH) (Ref. 37) & 1.1154 & 1.759 \\
Weeks-Chandler-Andersen (WCA) (Ref. 38) & 1.1137 & {$\left[0.72157+0.04561 \rho^{*}-0.07468 \rho^{* 2}+0.12344 \rho^{* 3}\right]^{-2}$} \\
Lado (LWCA) (Ref. 14) & 1.1152 & {$\left[0.73454+0.10250 \rho^{*}-0.12960 \rho^{* 2}+0.15976 \rho^{* 3}\right]^{-2}$} \\
Ben-Amotz-Herschbach (BAH) (Ref. 39) & 1.1532 & 0.527 \\
\hline \hline
\end{tabular}


TABLE IV. Comparison of EHSD values predicted from $C_{1}$ with those from the models in Table III.

\begin{tabular}{|c|c|c|c|c|c|c|c|}
\hline \multicolumn{2}{|c|}{ LJ state } & \multicolumn{4}{|c|}{$\sigma^{*}$ values from the models in Table III } & \multicolumn{2}{|c|}{ Voronoi analysis } \\
\hline$T^{*}$ & $\rho^{*}$ & BH (Ref. 37) & WCA (Ref. 38) & LWCA (Ref. 14) & BAH (Ref. 39) & $C_{1}^{\mathrm{a}}$ & $\sigma^{*}$ \\
\hline 0.7408 & 0.8350 & 1.0262 & 1.0224 & 1.0200 & 1.0123 & 14.47 & 1.0318 \\
\hline 0.8230 & 0.8010 & 1.0226 & 1.0193 & 1.0169 & 1.0074 & 14.55 & 1.0295 \\
\hline 1.0649 & 0.7000 & 1.0134 & 1.0113 & 1.0090 & 0.9952 & 14.77 & 1.0246 \\
\hline 1.0662 & 0.8210 & 1.0133 & 1.0096 & 1.0068 & 0.9951 & 14.58 & 1.0143 \\
\hline 1.0845 & 0.7690 & 1.0127 & 1.0097 & 1.0072 & 0.9943 & 14.67 & 1.0188 \\
\hline 2.5655 & 0.4000 & 0.9775 & 0.9782 & 0.9760 & 0.9497 & 15.24 & 0.9884 \\
\hline 2.7371 & 0.3000 & 0.9746 & 0.9758 & 0.9739 & 0.9461 & 15.33 & 0.9830 \\
\hline 2.7584 & 0.7195 & 0.9742 & 0.9718 & 0.9686 & 0.9457 & 14.91 & 0.9735 \\
\hline 3.2617 & 0.9200 & 0.9666 & 0.9600 & 0.9558 & 0.9364 & 14.70 & 0.9540 \\
\hline 3.8833 & 0.9900 & 0.9583 & 0.9496 & 0.9449 & 0.9267 & 14.66 & 0.9398 \\
\hline
\end{tabular}

${ }^{\mathrm{a}}$ Averaged for 1000 configurations of $256 \mathrm{LJ}$ molecules, with PBC.

control parameter. In the dilute limit, the random structures produced at any success rate are identical to the thermodynamic structures. Above the freezing density, higher success rates produce more random structures, and the limit of near $100 \%$ success rate gives the maximally random structures. The neighbor coordination numbers $C_{n}$ analyzed here, $C_{2}$ and $C_{3}$ in two dimensions and $C_{1}, C_{2}$, and $C_{3}$ in three dimensions, have an inflection at the freezing transition for thermodynamic structures, but the maximally random structures do not have an inflection point around the freezing density. The first nearest neighbor coordination number $C_{1}$ for random hard-sphere structures produced by our algorithm agrees with the dense random packing experimental results of Finney. ${ }^{11}$

For a hard-rod system $g_{n}(r)$ is exactly known. ${ }^{26}$ For the 2D Poisson tessellation $f_{1}(r)$ (Ref. 24) and $P_{n}$ (Ref. 30) are exactly known. For any nondegenerate $2 \mathrm{D}$ tessellation with periodic boundary conditions, $C_{1}=6$ exactly. ${ }^{23,24}$ For the $2 \mathrm{D}$ Poisson tessellation, we report $C_{2} \approx 13.698$ and $C_{3} \approx 22.94$. For the $3 \mathrm{D}$ Poisson tessellation, $C_{1}=\frac{48}{35} \pi^{2}+2 \approx 15.5354$ exactly, ${ }^{23}$ and we report $C_{2} \approx 69.8$.

On freezing, the hard-disk coordination numbers $C_{n}$ (for $n>1)$ decrease sharply to the regular hexagonal lattice values $\left(C_{n}\right)_{\text {reg }}=6 n$. A sharp rise in the hexagon population and a sharp drop in the population of the other polygons mark the onset of hard-disk freezing transition. The hard-disk random structures have a slow rise in the hexagon population with increasing density, and the pentagon and heptagon populations remain nonzero as dense random packing is approached. In dense hard-disk structures, both thermodynamic and random, the pentagon and heptagon populations seem identical.

For the perfect fcc lattice $C_{1}=12$ and $C_{2}=42$. However, due to topological instability, ${ }^{12}$ a slightly perturbed fcc lattice has Voronoi polyhedra with faces $12-18$, with the mean at 14. This increase is achieved by forming tiny quadrilateral faces with a few second neighbors, thereby promoting them into first neighbors. This promotion manifests as a secondary peak in $g_{1}(r)$. Thus, on freezing transition the hard-sphere $C_{1}$ is close to 14 rather than 12 . We have demonstrated that this result is consistent with thermodynamic data. On freezing transition, even though there is a rise in the rhombic dodecahedron population, the population of the polyhedra with 13-18 faces remains significant. In hard-sphere random structures, the dodecahedron population decreases with increasing density. These results show the significant differences between the hard-sphere and hard-disk microstructures.

We show that the Voronoi neighbor statistic $C_{1}$ is useful in estimating the effective hard-sphere diameter of soft potential fluids. By matching the $C_{1}$ of the Lennard-Jones fluid configurations with that of the thermodynamic hard-sphere fluid at some packing fraction, we are able to estimate the effective hard-sphere diameter of the Lennard-Jones fluid within 2\% deviation from the theoretical results of Barker-Henderson $^{37}$ and Weeks-Chandler-Andersen ${ }^{38}$ and the modification by Lado. ${ }^{14}$ This statistical-geometric approach is elegant because it does not employ integral criteria for the repulsive part of the potential (as in the abovementioned theories) nor utilize property data fitting (as in the empirical correlations).

\section{APPENDIX: TOPOLOGICAL INSTABILITY OF FCC LATTICE}

The Rhombic dodecahedron (Fig. 17) is the Wigner-

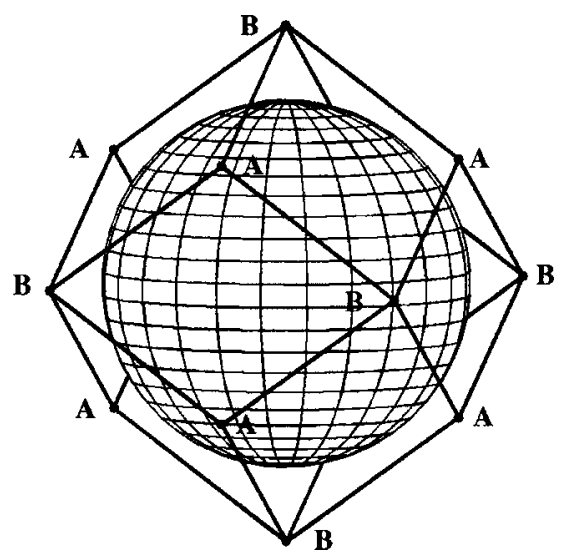

FIG. 17. Rhombic dodecahedron is the Wigner-Seitz or Voronoi cell for the perfect fcc lattice. 


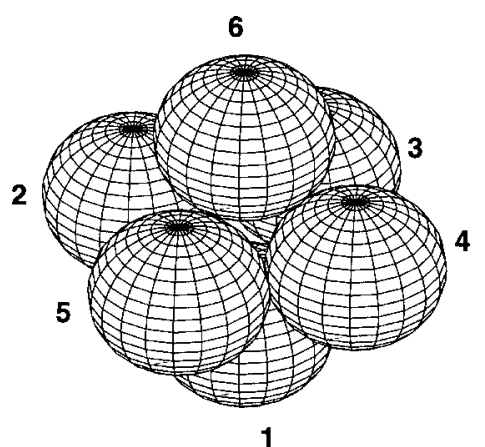

1

FIG. 18. The octahedron formed by the spheres sharing a type- $B$ vertex. For sphere 1 , the spheres $2-5$ are first neighbors, while sphere 6 is the second neighbor, if the lattice is nonperturbed. Small perturbations can promote sphere 6 into a first neighbor for sphere 1 .

Seitz cell or the Voronoi polyhedron for the perfect fcc lattice. It has 12 identical rhombic faces and 14 vertices. Its vertices are classified into two types based on their connectivity. Vertex type $A$ has four edges incident on it, with three edges from the given cell and another edge from the neighboring cells. Vertex type $B$ has eight edges incident on it, with four edges from the given cell and four from the neighboring cells. A rhombic dodecahedron has eight type- $A$ vertices and six type- $B$ vertices. A type- $B$ vertex is shared by six spheres, the centers of which form an octahedron (Fig. 18). The type- $B$ vertices are topologically unstable and, on perturbation, form additional edges leading to pentagonal or hexagonal faces or form an additional tiny quadrilateral face with a second neighbor sphere, thereby promoting it into a first neighbor (Fig. 19). When an additional face is formed, it is perpendicular to the diagonal of the octahedron formed by the central sphere and the sphere getting promoted as a first neighbor. Among all possible infinitesimal perturbations of

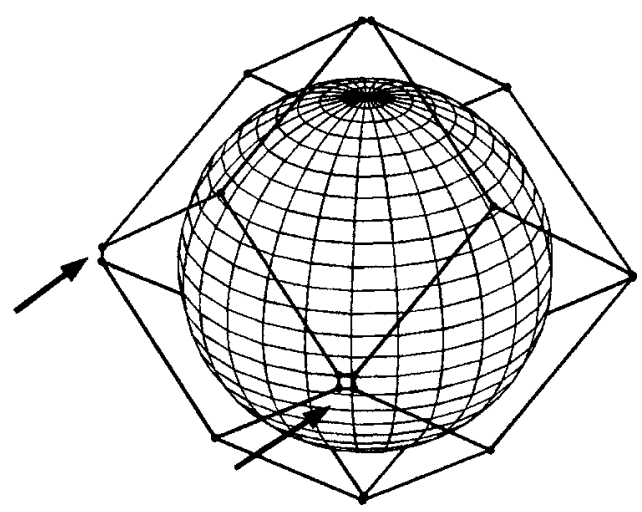

FIG. 19. A Voronoi cell from a perturbed fcc lattice having pentagonal or hexagonal faces due to the formation of additional edges or having an additional face formed with an earlier second neighbor. the lattice shown in Fig. 18, there will be an equal number of perturbations leading to the formation of additional faces between the pairs of spheres $(1,6),(2,4)$, or $(3,5)$. Hence, the probability that an additional face is formed between one of the pairs is $\frac{1}{3}$. Thus, the average number of faces equals 12 plus the number of type- $B$ vertices times the probability that a type- $B$ vertex forms an additional face $\left(12+6 \times \frac{1}{3}=14\right)$. This was proven by Troadec et $a l .{ }^{12}$ (further details are therein).

${ }^{1}$ G. Voronoi, J. Reine Angew. Math. 134, 198 (1908).

${ }^{2}$ D. C. Rapaport, Mol. Phys. 48, 23 (1983).

${ }^{3}$ F. Aurenhammer, ACM Comput. Surv. 23, 345 (1991)

${ }^{4}$ A. Okabe, B. Boots, and K. Sugihara, Spatial Tessellations: Concepts and Applications of Voronoi Diagrams (Wiley, New York, 1992).

${ }^{5}$ G. Schliecker, Adv. Phys. 51, 1319 (2002).

${ }^{6}$ B. N. Boots, Metallography 20, 231 (1982).

${ }^{7}$ H. X. Zhu, S. M. Thorpe, and A. H. Windle, Philos. Mag. A 81, 2765 (2001)

${ }^{8}$ L. Oger, A. Gervois, J. P. Troadec, and N. Rivier, Philos. Mag. B 74, 177 (1996).

${ }^{9}$ L. V. Woodcock, J. Chem. Soc., Faraday Trans. 2 72, 1667 (1976).

${ }^{10}$ S. Torquato, T. M. Truskett, and P. G. Debenedetti, Phys. Rev. Lett. 84, 2064 (2000).

${ }^{11}$ J. L. Finney, Proc. R. Soc. London, Ser. A 319, 479 (1970).

${ }^{12}$ J. P. Troadec, A. Gervois, and L. Oger, Europhys. Lett. 42, 167 (1998).

${ }^{13}$ C. Silva, H. Liu, and E. A. Macedo, Ind. Eng. Chem. Res. 37, 221 (1998).

${ }^{14}$ F. Lado, Mol. Phys. 52, 871 (1984).

${ }^{15}$ G. Y. Onoda and E. G. Liniger, Phys. Rev. Lett. 64, 2727 (1990).

${ }^{16}$ B. J. Alder and T. E. Wainwright, Phys. Rev. 127, 359 (1962).

${ }^{17}$ W. G. Hoover and F. H. Ree, J. Chem. Phys. 49, 3609 (1968).

${ }^{18}$ J. G. Berryman, Phys. Rev. A 27, 1053 (1983).

${ }^{19}$ E. L. Hinrichsen, J. Feder, and T. Jossang, Phys. Rev. A 41, 4199 (1990).

${ }^{20}$ A. Rahman, J. Chem. Phys. 45, 2585 (1966).

${ }^{21}$ N. L. Lavrik and V. P. Voloshin, J. Chem. Phys. 114, 9489 (2001).

${ }^{22} \mathrm{~A} 2 \mathrm{D}$ Voronoi vertex (and hence the tessellation) is degenerate if four edges are incident on it, as in a simple cubic lattice of hard-disks, with square cells. A 3D Voronoi vertex is degenerate if eight edges are incident on it, as in a fcc lattice of hard-spheres, with rhombic dodecahedron cells.

${ }^{23}$ J. L. Meijering, Philips Res. Rep. 8, 270 (1953).

${ }^{24}$ R. Collins, J. Phys. C 1, 1461 (1968).

${ }^{25}$ T. Ogawa and M. Tanemura, Prog. Theor. Phys. 51, 399 (1974).

${ }^{26}$ I. Z. Fisher, Statistical Theory of Liquids (The University of Chicago Press, Chicago, 1964).

${ }^{27}$ L. Tonks, Phys. Rev. 50, 955 (1936).

${ }^{28}$ D. K. Stillinger, F. H. Stillinger, S. Torquato, T. M. Truskett, and P. G. Debenedetti, J. Stat. Phys. 100, 49 (2000).

${ }^{29}$ J. M. Drouffe and C. Itzykson, Nucl. Phys. B 235, 45 (1984).

${ }^{30}$ P. Calka, Adv. Appl. Probab. 35, 863 (2003).

${ }^{31}$ D. A. Young and B. J. Alder, J. Chem. Phys. 70, 473 (1979).

${ }^{32}$ R. Jullien, P. Jund, D. Caprion, and D. Quitmann, Phys. Rev. E 54, 6035 (1996).

${ }^{33}$ L. Boltzmann, Lectures on Gas Theory, translated by S. G. Brush (University of California Press, Berkley, 1964), p. 169.

${ }^{34}$ J. G. Kirkwood and E. M. Boggs, J. Chem. Phys. 10, 394 (1942).

${ }^{35}$ L. Verlet, Phys. Rev. 165, 201 (1968).

${ }^{36}$ D. M. Heyes, Phys. Rev. B 37, 5677 (1988).

${ }^{37}$ J. A. Barker and D. Henderson, Rev. Mod. Phys. 48, 587 (1976).

${ }^{38}$ D. Chandler, J. D. Weeks, and H. C. Andersen, Science 220, 787 (1983).

${ }^{39}$ D. Ben-Amotz and D. R. Herschbach, J. Phys. Chem. 94, 1038 (1990). 